\title{
Can a Consumer Ultraviolet Disinfection Device be Repurposed for N95 Mask Decontamination during the COVID-19 pandemic
}

\author{
Robert Lambkin-Williams ${ }^{1}$ \\ ${ }^{1}$ VirologyConsult Ltd
}

August 3, 2020

\begin{abstract}
Abstract. The reuse of N95 masks has been forced on small community-based hospitals, dentists, nursing, and care homes, amongst others, due to the desperate shortage of personal protective equipment globally during the COVID-19 pandemic. The CDC issued guidance on the reuse of N95 masks due to this shortage. However, large scale ultraviolet disinfection equipment would not be practical in these circumstances, we, therefore, investigated the efficacy of a small, mobile, benchtop device with the potential to disinfect N95 masks from SARS-CoV-2 using ultraviolet irradiation. The device tested was the Lumin LM3000 System manufactured by 3B Medical. We found the Lumin LM3000 to be effective against enveloped viruses, including coronavirus, non-enveloped viruses, gram-negative and gram-positive bacteria and spores with reductions greater than 99.9 per centAbstract content goes here
\end{abstract}

\section{Hosted file}

Can a Consumer Ultraviolet Disinfection Device be Repurposed for N95 Mask Decontamination during the CO available at https://authorea.com/users/323495/articles/473570-can-a-consumer-ultravioletdisinfection-device-be-repurposed-for-n95-mask-decontamination-during-the-covid-19pandemic

\section{Hosted file}

Supplementary E Microchem- S aureus K pneumoiae -NG14592.pdf available at https: //authorea.com/users/323495/articles/473570-can-a-consumer-ultraviolet-disinfectiondevice-be-repurposed-for-n95-mask-decontamination-during-the-covid-19-pandemic

\section{Hosted file}

Supplementary D Stearothermophilus CPAP Accessories.pdf available at https://authorea. com/users/323495/articles/473570-can-a-consumer-ultraviolet-disinfection-device-berepurposed-for-n95-mask-decontamination-during-the-covid-19-pandemic

\section{Hosted file}

Supplementary C 3B Products Lumin UV Mask Study Adenovirus 2070420 BCS ID 2006241r.pdf available at https://authorea.com/users/323495/articles/473570-can-a-consumer-ultravioletdisinfection-device-be-repurposed-for-n95-mask-decontamination-during-the-covid-19pandemic

\section{Hosted file}

Supplementary B Lumin Influenza H1N1 Study.pdf available at https://authorea.com/users/ 323495/articles/473570-can-a-consumer-ultraviolet-disinfection-device-be-repurposed-for- 
n95-mask-decontamination-during-the-covid-19-pandemic

\section{Hosted file}

Supplemnetary A Human Coronavirus on N95 Microchem ASTM E1053 Report NG14983V2.pdf available at https://authorea.com/users/323495/articles/473570-can-a-consumer-ultravioletdisinfection-device-be-repurposed-for-n95-mask-decontamination-during-the-covid-19pandemic

\section{Hosted file}

Supplementary F Microchem- S aureus K pneumoiae -NG14592.pdf available at https: //authorea.com/users/323495/articles/473570-can-a-consumer-ultraviolet-disinfectiondevice-be-repurposed-for-n95-mask-decontamination-during-the-covid-19-pandemic

\section{Hosted file}

Supplementary H Microchem- S- Enterica-NG14813-V2-SE10708.pdf available at https://authorea . com/users/323495/articles/473570-can-a-consumer-ultraviolet-disinfection-device-berepurposed-for-n95-mask-decontamination-during-the-covid-19-pandemic

\section{Hosted file}

Supplementary I Mesa- CPAP Accessories B pumilus.pdf available at https://authorea. com/users/323495/articles/473570-can-a-consumer-ultraviolet-disinfection-device-berepurposed-for-n95-mask-decontamination-during-the-covid-19-pandemic 